\title{
Synthesis of Nanowire-Shaped Silver by Polyol Process of Sodium Chloride
}

\author{
Nguyen V. Nghia ${ }^{1, *}$, Nguyen N. K. Truong ${ }^{1}$, Nguyen M. Thong ${ }^{2}$, Nguyen P. Hung ${ }^{2}$ \\ ${ }^{1}$ Department of Physics, Quy Nhon University, Quy Nhon City, Binh Dinh Pro., Vietnam \\ ${ }^{2}$ Department of Chemistry, Quy Nhon University, Quy Nhon City, Binh Dinh Pro., Vietnam \\ nguyenvannghia@qnu.edu.vn
}

\begin{abstract}
Nanowire-shaped silver have been synthesized by the polyol process in ethylene glycol as a reductant, polyvinylpyrrolidone (PVP) as a stabilizer, using a microwave technique. The products were characterized by transmission electron microscopy (TEM). The presence of sodium chloride in the polyol reduction of silver nitrate facilitated the production of silver nanowires. These wires were formed quickly (in approximately 3 minutes microwave heating). It was found that morphologies and sizes of silver nanostructures depended strongly on such experimental parameters as concentrations of $\mathrm{PVP}, \mathrm{NaCl}, \mathrm{AgNO}_{3}$, and heating time. The chloride ion was necessary to synthesize nanowire-shaped silver, and the sodium chloride likely controlled the rate of silver(I) reduction and initial seed formation.
\end{abstract}

Keywords Silver Nanostructures, Chloride Anion, Polyvinylpyrrolidone, Microwave Heating, Polyol Method

\section{Introduction}

Among all the nanowire materials, the synthesis of silver nanowires has been and continues to be an area of active research because of their wide applications in catalysts, scanning probes, and various kinds of electronic and photonic nanodevices. Microwave-polyol method is a promising route for rapid preparation of metallic nanomaterials[1]. When microwave was irradiated into the mixture of $\mathrm{AgNO}_{3} / \mathrm{NaCl} / \mathrm{PVP}$ in ethylene glycol solution, anisotropic Ag nanarods and nanowires were produced preferentially. In this work, we examined the dependence of shape, size of silver nanostructures on such experimental parameters as concentrations of PVP, $\mathrm{NaCl}, \mathrm{AgNO}_{3}$, and heating time. The changes of shapes and sizes of silver nanostructures were observed by using transmission electron microscope (TEM). Possible formation mechanism of nanorods, nanowires, spherical and cubic nanoparticles under microwave (MW) irradiation in the presence of sodium chloride was discussed.

\section{Experimental}

A microwave oven (Sanyo, 2.45Ghz, 800W) was modified by installing a condenser through holes of the ceiling. Polyvinyl pyrrolidone (PVP, Mw=360K Sigma Aldrich, 99.9\%) was homogeneously dispersed into ethylene glycol

* Corresponding author:

nguyenvannghia@qnu.edu.vn (Nguyen V. Nghia)

Published online at http://journal.sapub.org/ijmc

Copyright (C) 2012 Scientific \& Academic Publishing. All Rights Reserved
(EG, China, 96\%) in a $100 \mathrm{~mL}$ pyrex becher. Then silver nitrate $\mathrm{AgNO}_{3}$, sodium chloride $\mathrm{NaCl}$ were added into the solution and stirred until the $\mathrm{AgNO}_{3}$ and $\mathrm{NaCl}$ dissolved completely. The resulted solutions were heated in the microwave oven at $400 \mathrm{~W}$ for various concentrations of $\mathrm{AgNO}_{3}$, PVP, $\mathrm{NaCl}$, and heating time. The synthesized silver colloidal solutions were characterized by analytical technique TEM (JEM-1400).

\section{Results and Discussion}

\subsection{Effects of Heating Time}

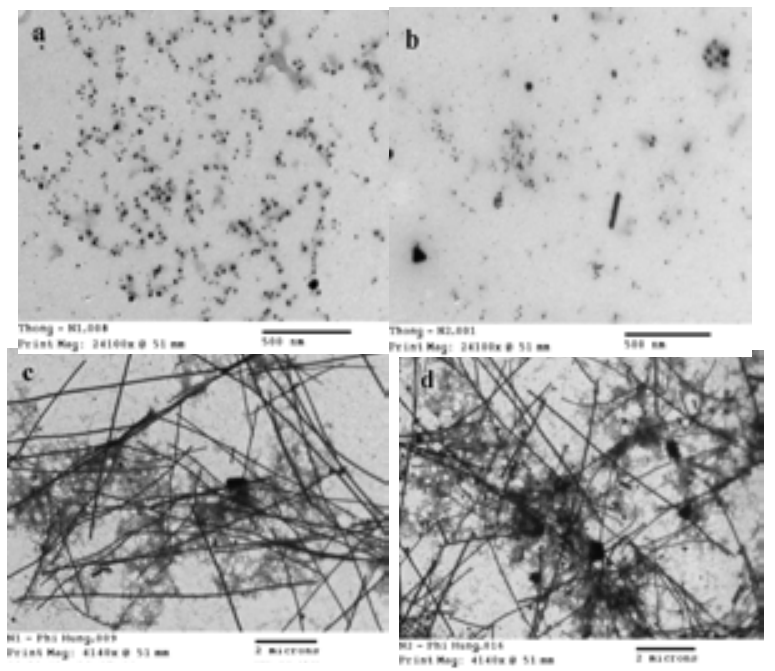

Figure 1. TEM images of Ag nanostructures synthesized from $\mathrm{AgNO}_{3}$ / $\mathrm{NaCl} / \mathrm{PVP} / \mathrm{EG}$ with the microwave irradiation time $1.5 \mathrm{~min}$ (a), $2 \mathrm{~min}$ (b), $3.5 \min (\mathrm{c})$, and 5 min (d) 
Figures 1(a)-(d) show TEM images of Ag nanostructures synthesized from $\mathrm{AgNO}_{3} / \mathrm{NaCl} / \mathrm{PVP} / \mathrm{EG}$ after MW irradiation for $1.5,2,3.5$ and 5 minutes, respectively. At the shortest irradiation time 1.5 minutes, only spherical nanoparticles with a diameter of about $15-25 \mathrm{~nm}$ are produced (figure 1a). When increasing irradiation time, anisotropic nanoparticles appear and they are grown to nanorods and nanowires, the same results as considered in[2]. At an irradiation time of 3.5 minutes, nanowires are formed. We see little change in the TEM images after MW irradiation for 3.5 minutes, it proves that the $\mathrm{Ag}^{+}$ions reduction was occurred completely. Therefore, we fixed MW time 3.5 minutes to carry out further research.

\subsection{Effects of PVP Concentration}

To test the effects of PVP concentration to morphology of Ag nanostructures, nanosized Ag colloids are synthesized at different concentrations of PVP. Figures 2 (a)-(c) show the typical TEM images of nanosized Ag colloids synthesized from $\mathrm{AgNO}_{3}(60 \mathrm{mM}) / \mathrm{NaCl}(3 \mathrm{mM}) / \mathrm{PVP} / \mathrm{EG}$ with PVP concentration of $25 \mathrm{mM}, 50 \mathrm{mM}, 200 \mathrm{mM}$, respectively. At the the lowest PVP concentration of $25 \mathrm{mM}$, aggregation occurs so that large spherical particles are major products. When the PVP concentration increased to $50 \mathrm{mM}$, the products of nanowires increased considerably. But with increasing PVP concentration too high (200 mM), highly crystalline Ag cubes and triangular bipyramids are preferentially. From the above results, we can conclude that the concentration of PVP has a significant effect on the shape and size of Ag nanostructured.
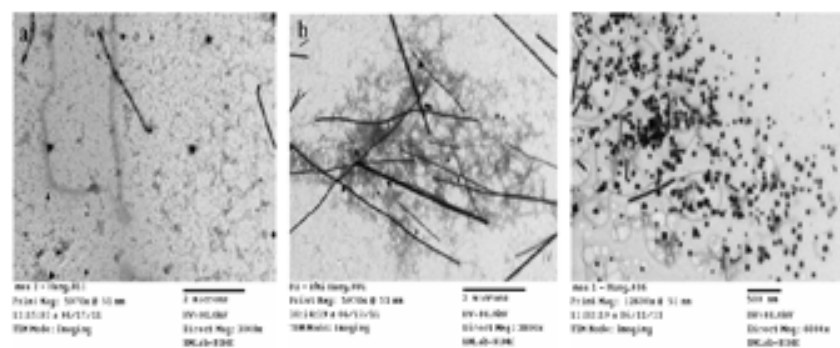

Figure 2. TEM images of Ag nanostructures synthesized from $\mathrm{AgNO}_{3} /$ $\mathrm{NaCl} / \mathrm{PVP} / \mathrm{EG}$ with PVP concentrations of $25 \mathrm{mM}(\mathrm{a}), 50 \mathrm{mM}(\mathrm{b}), 200$ $\mathrm{mM}(\mathrm{c})$

\subsection{Effects of $\mathrm{AgNO}_{3}$ Concentration}

Figures 3 (a)-(d) show the typical TEM images of $\mathrm{Ag}$ nanostructures synthesized from $\mathrm{AgNO}_{3} / \mathrm{NaCl}(3 \mathrm{mM}) / \mathrm{PVP}$ $(50 \mathrm{mM}) / \mathrm{EG}$ at the concentration of $\mathrm{AgNO}_{3}$ of $15 \mathrm{mM}, 30$ $\mathrm{mM}, 60 \mathrm{mM}$, and $90 \mathrm{mM}$, respectively. From the results of TEM images, we see the shape and size of products depend strongly on the concentration of $\mathrm{AgNO}_{3}$. At the low concentration of $\mathrm{AgNO}_{3} 15 \mathrm{mM}$, cubes and triangular bipyramids are dominant products. In contrast, the yields of 1-D (rods and wires) products increase with increasing the concentration of $\mathrm{AgNO}_{3}$. At the highest concentration of $\mathrm{AgNO}_{3}$ $90 \mathrm{mM}$, the yields of 1 -D products are more than $80 \%$.
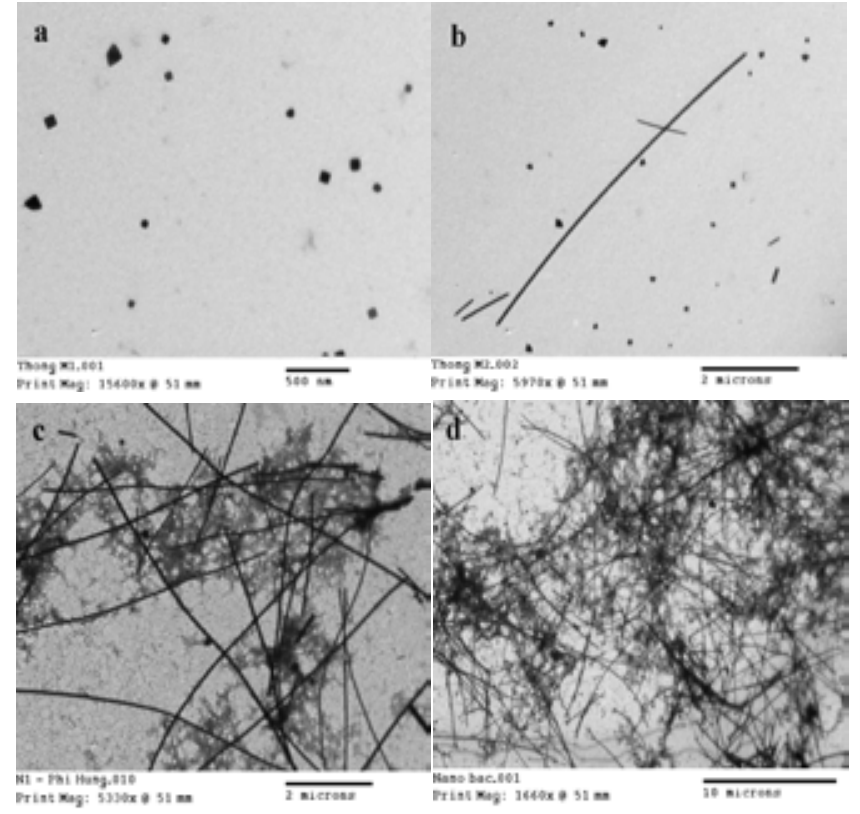

Figure 3. TEM images of Ag nanostructures are synthesized from $\mathrm{AgNO}_{3} / \mathrm{NaCl}(3 \mathrm{mM}) / \mathrm{PVP}(50 \mathrm{mM}) / \mathrm{EG}$ at the concentrations of $\mathrm{AgNO}_{3}$ of $15 \mathrm{mM}$ (a), $30 \mathrm{mM}$ (b), $60 \mathrm{mM}$ (c), $90 \mathrm{mM}$ (d)
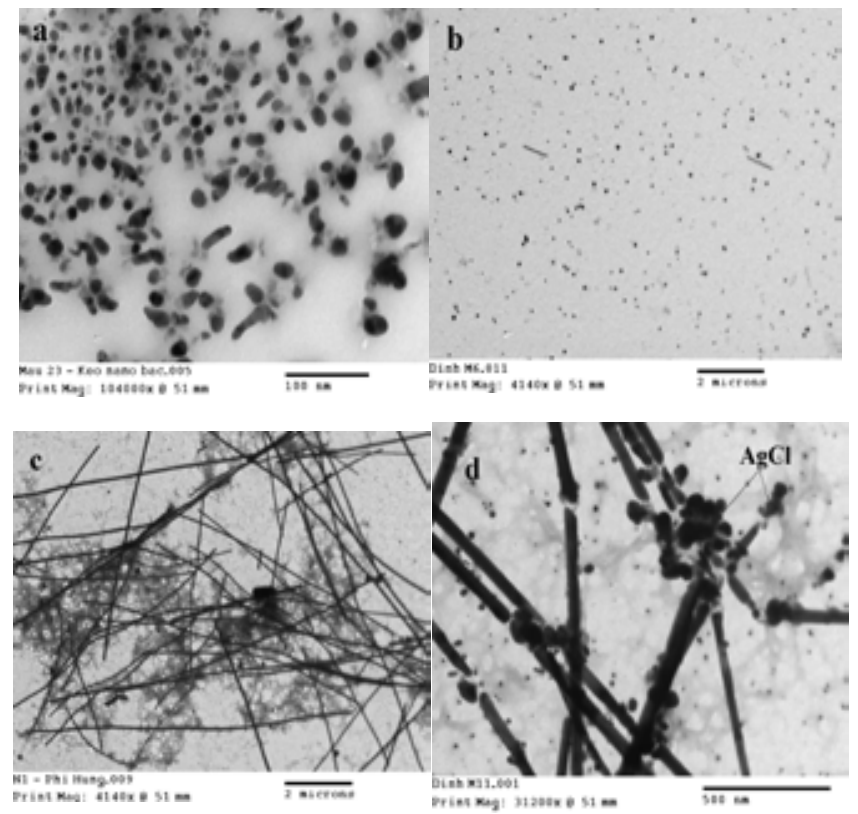

Figure 4. TEM images of Ag nanostructures are synthesized from $\mathrm{AgNO}_{3} / \mathrm{NaCl} / \mathrm{PVP} / \mathrm{EG}$ at $\mathrm{NaCl}$ concentrations of $0 \mathrm{mM}$ (a), $1 \mathrm{mM}$ (b), 3 $\mathrm{mM}$ (c), 10 mM (d)

\subsection{Effects of NaCl Concentration}

Figures 4 (a)-(d) show the typical TEM images of $\mathrm{Ag}$ nanocolloid are synthesized from $\mathrm{AgNO}_{3}(60 \mathrm{mM}) / \mathrm{NaCl} /$ PVP(50 mM)/EG with $\mathrm{NaCl}$ concentrations of $0 \mathrm{mM}$ (a), 1 $\mathrm{mM}$ (b), $3 \mathrm{mM}$ (c), and $10 \mathrm{mM}$ (d), respectively. In the absence of $\mathrm{NaCl}$, the main products are the spherical silver nanoparticles (96\%) besides there is a small rod-shaped form. At room temperature, the addition of $\mathrm{NaCl} 1 \mathrm{mM}$ in a solution of $\mathrm{AgNO}_{3}$ leads to a very small amount of soluble $\mathrm{AgCl}$ formed due to the small solubility constant of AgCl. Along 
with the increase of reaction temperature, $\mathrm{AgCl}$ is dissolved as $\mathrm{Ag}^{+}$and $\mathrm{Cl}^{-}$, then $\mathrm{Ag}^{+}$ions is reduced to $\mathrm{Ag}^{0}$, seed formation and crystal growth take place. Because twinning probability is low, single crystal cubes and single-twin bipyramid are produced preferentially. When $\mathrm{NaCl}$ concentration increased, the amount of $\mathrm{AgCl}$ is formed more and the standard electrode potential of $\mathrm{AgCl} / \mathrm{Ag}=+0.2233$ (compared to the standard hydrogen electrode) lower electrode of the $\mathrm{Ag}^{+} / \mathrm{Ag}=+0.799$ (compared to the standard hydrogen electrode), most atoms $\mathrm{Ag}^{0}$ not formed from $\mathrm{AgCl}$ that $\mathrm{Ag}^{+}$ ions freedom[3]. In general, in the presence of $\mathrm{NaCl}$, the $\mathrm{Ag}$ product obtained is a mixture of nanorods, nanowires, triangular bipyramids, spherical and cubic nanoparticles. From the results of TEM images, we see at a concentration of $\mathrm{NaCl}$ $3 \mathrm{mM}$ are optimum conditions for synthesis of silver nanowires. However, at higher concentrations of $\mathrm{NaCl} 10$ $\mathrm{mM}$, besides 1D (nanorods and nanowires) products, amorphous AgCl particles also appeared[4].

\subsection{Growth mechanisms of Ag nanostructures}

The reaction in the formation of Ag nanocolloid in ethylene glycol can be described as follows[5]:

At high temperature dehydrated process occurs:

$$
\mathrm{CH}_{2} \mathrm{OH}-\mathrm{CH}_{2} \mathrm{OH} \rightarrow \mathrm{CH}_{3} \mathrm{CHO}+\mathrm{H}_{2}
$$

Then $\mathrm{Ag}^{+}$ions coordinate bond with NCO groups of $\mathrm{PVP}[6]$ are reduced:

$$
2 \mathrm{CH}_{3} \mathrm{CHO}+2 \mathrm{Ag}^{+} \rightarrow 2 \mathrm{Ag}+2 \mathrm{H}^{+}+\mathrm{CH}_{3} \mathrm{COCOCH}_{3} \quad \text { (2) }
$$

Also in reaction (1), according to the authors[7] while reaction:

$$
2 \mathrm{HOCH}_{2}-\mathrm{CH}_{2} \mathrm{OH}+\mathrm{O}_{2} \rightarrow 2 \mathrm{HOCH}_{2} \mathrm{CHO}+2 \mathrm{H}_{2} \mathrm{O}
$$

According to these authors, the temperature of reaction was above $150{ }^{\circ} \mathrm{C}$, the reaction formation (3), was producted glycolaldehyde (GA), GA reduces more powerfully than that of acetic aldehyde.

Polymer PVP plays a role of developmental control Ag particle size and form Ag products with nanometer size.

Growth mechanism of Ag nanostructures under MWpolyol method in the presence of PVP stabilizer and sodium chloride can be described in figure 5[4]. A small amount of sodium chloride is added to act as intermediates to produce the seed in the formation of Ag nano structures. The seeds play an important role in shaping the original shape of $\mathrm{Ag}$ nanoparticles with the structure "shell-core": "Ag-seed". While PVP has an important role in taking form the final shape of Ag nanostructures. A low concentrations of seeds and a high concentrations of PVP are favourable for the growth of cubic crystals and the polygonal nanoplates. On other hand, combination of a medium concentration of seeds with low concentrations of PVP are favourable for the growth of nanorods, nanowires and nanosheets[8,9].

The presence of trace amounts of chloride ion also acts an important role in the formation of Ag nanowires in ethylene glycol solvent by microwave method. Firstly, it provides electrostatic stabilization for the initial formed silver seeds. Secondly, chloride ion can reduce the concentration of free $\mathrm{Ag}^{+}$ions in solution through the formation of $\mathrm{AgCl}$ nanocrystallites and supply a low release process of $\mathrm{Ag}^{+}$ which facilitates the high-yield formation of the thermodynamically more stable multiple-twined particles (MTPs) required for wire growth. MTPs consist of ten $\{111\}$, these $\{111\}$ faces must remain as active top growth surfaces, while the growth of five side $\{100\}$ faces must be suppressed by preferential absorption of PVP to $\{100\}$ faces. Therefore, Ag atoms are preferentially deposited onto $\{111\}$ surface, leading to the anisotropic growth of silver nanowires[10]. The growth rates to the two opposite $<110>$ directions are nearly the same and the diameter of nanorod is close to the diameter of MTP[9] (figure 6).

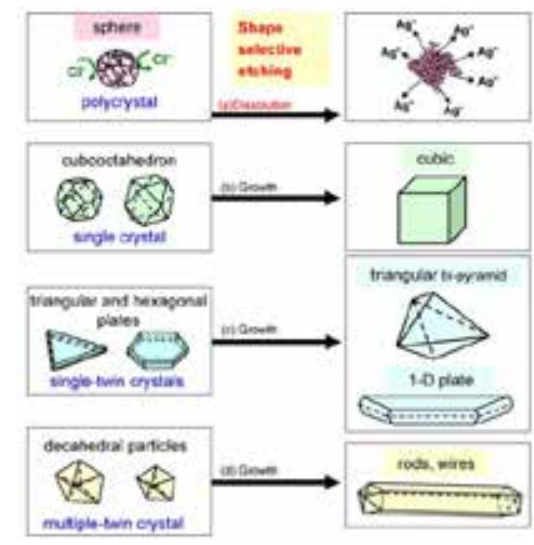

Figure 5. Growth mechanism of Ag nano-structure products from seed particles
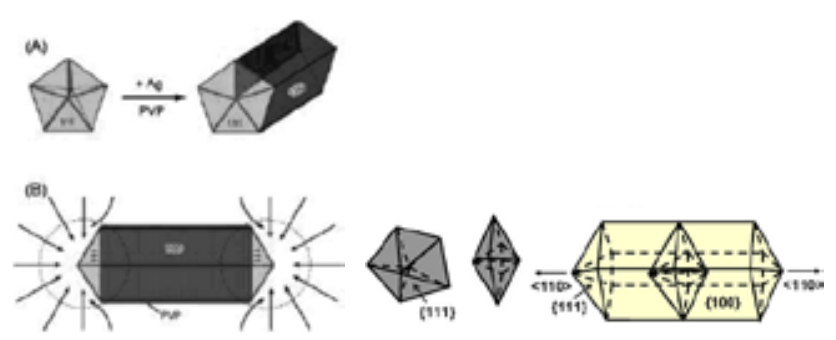

Figure 6. Growth mechanism of nanorod and nanowire[13]

When low PVP concentrations and short heating time, thin Ag nanosheets having $\{111\}$ faces on the top and bottom surfaces are formed. When the nanosheets are grown by expanding fcc (face centered cubic) layers to three side $<110>$ directions, PVP must attach selectively to the $\{111\}$ faces of silver. Thus the selective attachment of PVP occurs not only to the $\{100\}$ faces having the second low surface energy but also to the $\{111\}$ faces having the lowest surface energy[11,12], depending on the morphologies of the product and the experimental conditions.

On the other hand, at high PVP concentrations, PVP cover fully "shell-core" seeded nanoparticles. Therefore, spherical and highly symmetrical cubic crystals will be prepared preferentially. The formation of cubic crystals fully surrounded by $\{100\}$ faces, indicates that PVP attach preferentially to the $\{100\}$ faces $[4,9]$.

\section{Conclusions}


In conlusion, we have succeeded in rapid preparation of such anisotropic Ag nanostructures within a few minutes as nanorods, nanowires, and nanocubes by using microwave-polyol method. The shapes and sizes of Ag nanostructures depends strongly on such experimental parameters as concentration of $\mathrm{AgNO}_{3}, \mathrm{PVP}, \mathrm{NaCl}$ and heating time. At low concentration of PVP (50 mM), medium concentration of $\mathrm{NaCl}(3 \mathrm{mM})$ and heating time of 3 minutes are favourable for the formation of Ag nanorods and nanowires. The synthesis of Ag nanowires in the presence of $\mathrm{NaCl}$ is a new point of this work. The advantages here are simple equipment and process, the much cheaper of $\mathrm{NaCl}$ compared to $\mathrm{H}_{2} \mathrm{PtCl}_{6}$ in other previous publications.

\section{ACKNOWLEDGEMENTS}

This work was supported in part by the Grant-in-Aid for Scientific Research from the Ministry of Education and Training, Vietnam (Code B2012.28.47, 2012-2013).

\section{REFERENCES}

[1] M. Tsuji, M. Hashimoto, Y. Nishizawa (2005), 'Microwave-assisted synthesis of metallic nanostructures in solution', Chemistry - A European Journal, 11, pp.440-452.

[2] C. Murphy, N. R. Jana (2002), 'Controlling the aspect ratio of inorganic nanorods and nanowires’, Advanced Materials, 14, pp.80-82.

[3] X. Tang, M. Tsuji (2010), 'Syntheses of silver nanowires in liquid phase’, Nanowires Science and Technology, 89, p.402.

[4] M. Tsuji, K. Matsumoto, P. Jang, R. Matsuo, X. L. Tang, K. S. N. Kamarudin (2007), 'Roles of pt seeds and chloride anions in the preparation of silver nanorods and nanowires by microwave-polyol method', Colloids and Surfaces A: Physicochem. Eng. Aspects, 124, pp.76-80.

[5] K. Patel, S. Kapoor, D. P. Dave, T. Mukherjee (2005), 'Synthesis of nanosized silver colloids by microwave dielectric heating', J. Chem. Sci, 117, pp.53-60.

[6] P. Jiang, S. Y. Li, S. S. Xie, Y. Gao, L. Song (2004), 'Machinable long PVP-stabilized silver nanowires', Chemistry A European Journal, 10, pp.4817-4821.

[7] Y. Guangqing, W. Li, Z. Lei (2010), 'Recent research progress on preparation of gold nanotubes and Pt nanotubes from resultant silver nanowires and their application in conductive adhesive', Rev. adv. Mater. Sci, 24, pp.10-25.

[8] M. Tsuji, Y. Nishizawa, K. Matsumoto, N. Miyamae, T. Tsuji, M. Kubokawa (2006), 'Effects of chain length of polyvinylpyrrolidone for the synthesis of silver nanostructures by a microwave-polyol method', Materials Letters, 60, pp.834-838.

[9] M. Tsuji, Y. Nishizawa, K. Matsumoto, N. Miyamae, T. Tsuji, X. Zhang (2007), 'Rapid synthesis of silver nanostructures by using microwave-polyol method with the assistance of $\mathrm{Pt}$ seeds and polyvinylpyrrolidone', Colloids and Surfaces A: Physicochem. Eng. Aspects, 293, pp.185-194.

[10] Y. Sun, B. Gates, B. Mayers, Y. Xia (2002), 'Crystalline silver nanowires by soft solution processing', Nano Letters, 2 (2), pp.165-168.

[11] Y. Sun, Y. Xia (2002), 'Shape-controlled synthesis of gold and silver nanoparticles', Science, 298, pp.2176-2179.

[12] Z. L. Wang (2000), 'Transmission electron microscopy of shape-controlled nanocrystals and their assemblies', J. Phys. Chem. B, 106 (6), pp.1153-1175.

[13] Y. Sun, B. Mayers, T. Herricks, Y. Xia (2003), 'Polyol synthesis of uniform silver nanowires: a plausible growth mechanism and the supporting evidence', Nano Letters, 3(7), pp.955-960. 